Research Article

\title{
On Complex-Valued Triple Controlled Metric Spaces and Applications
}

\author{
Nabil Mlaiki $\left(\mathbb{D},{ }^{1}\right.$ Thabet Abdeljawad $\left(\mathbb{D},{ }^{1,2,3}\right.$ Wasfi Shatanawi $\left(\mathbb{D},{ }^{1}\right.$ Hassen Aydi $\mathbb{D}^{2,4,5}$ \\ and Yaé Ulrich Gaba ${ }^{6,7,8}$
}

\author{
${ }^{1}$ Department of Mathematics and General Sciences, Prince Sultan University, P.O. Box 66833, Riyadh 11586, Saudi Arabia \\ ${ }^{2}$ Department of Medical Research, China Medical University, Taichung 40402, Taiwan \\ ${ }^{3}$ Department of Computer Sciences and Information Engineering, Asia University, Taichung, Taiwan \\ ${ }^{4}$ Université de Sousse, Institut Supérieur d'Informatique et des Techniques de Communication, H. Sousse 4000, Tunisia \\ ${ }^{5}$ Department of Mathematics and Applied Mathematics, Sefako Makgatho Health Sciences University, Molotlegi St, Ga- \\ Rankuwa Zone 1, Ga-Rankuwa 0208, South Africa \\ ${ }^{6}$ Institut de Mathématiques et de Sciences Physiques (IMSP/UAC), Laboratoire de Topologie Fondamentale, Computationnelle et \\ Leurs Applications (Lab-ToFoCApp), BP 613 Porto-Novo, Benin \\ ${ }^{7}$ Quantum Leap Africa (QLA), AIMS Rwanda Centre, Remera Sector KN 3, Kigali, Rwanda \\ ${ }^{8}$ African Center for Advanced Studies (ACAS), P.O. Box 4477, Yaounde, Cameroon
}

Correspondence should be addressed to Thabet Abdeljawad; tabdeljawad@psu.edu.sa and Hassen Aydi; hassen.aydi@isima.rnu.tn Received 2 March 2021; Revised 31 March 2021; Accepted 12 April 2021; Published 28 April 2021

Academic Editor: Santosh Kumar

Copyright (C) 2021 Nabil Mlaiki et al. This is an open access article distributed under the Creative Commons Attribution License, which permits unrestricted use, distribution, and reproduction in any medium, provided the original work is properly cited.

In this manuscript, we introduce the concept of complex-valued triple controlled metric spaces as an extension of rectangular metric type spaces. To validate our hypotheses and to show the usability of the Banach and Kannan fixed point results discussed herein, we present an application on Fredholm-type integral equations and an application on higher degree polynomial equations.

\section{Introduction}

Since the breakthrough of Banach [1] in 1922, where he was able to show that a contractive mapping on a complete metric space has a unique fixed point, the field of fixed point theory has become an important research focus in the field of mathematics; see [2-6]. Due to the fact that fixed point theory has many applications in many fields of science, many researchers have been working on generalizing his result by either generalizing the type of contractions [7-10] or by extending the metric space itself $(b$-metric spaces $[11,12]$, controlled metric spaces [13], double controlled metric spaces [14], etc.). On the other hand, Azam et al. [15] defined complex-valued metric spaces and gave common fixed point results. Rao et al. [16] introduced the complex-valued $b$-metric spaces in the year 2013. Going in the same direction, recently, Ullah et al. [17] presented complex-valued extended $b$-metric spaces to extend the idea of extended $b$-metric spaces.

In this manuscript, following the path of the work done in [18], we extend complex-valued rectangular extended $b$ -metric spaces [19] to complex-valued triple controlled metric spaces. The layout of our manuscript is as follows. In the second section, we present some backgrounds along with the definition of complex-valued triple controlled metric spaces. In the third section, we prove some fixed point results in such spaces. In the fourth section, we present an application for our findings. In closing, we present two open questions.

\section{Preliminaries}

In what follows, owing to Azam et al. [15], we recall several notations and definitions which will be used in the sequel. 
Let $\mathbb{C}$ be the set of all complex numbers and $s_{1}, s_{2} \in \mathbb{C}$. The partial order on $\mathbb{C}$ is defined as $s_{1} \preceq s_{2}$ if and only if $\operatorname{Re}$ $\left(s_{1}\right) \leq \operatorname{Re}\left(s_{2}\right)$ and $\operatorname{Im}\left(s_{1}\right) \leq \operatorname{Im}\left(s_{2}\right)$. This implies that $s_{1} \preceq s_{2}$ if one of the below conditions is fulfilled:

(i) $\operatorname{Re}\left(s_{1}\right)=\operatorname{Re}\left(s_{2}\right), \operatorname{Im}\left(s_{1}\right)<\operatorname{Im}\left(s_{2}\right)$

(ii) $\operatorname{Re}\left(s_{1}\right)<\operatorname{Re}\left(s_{2}\right), \operatorname{Im}\left(s_{1}\right)=\operatorname{Im}\left(s_{2}\right)$

(iii) $\left(\operatorname{Re}\left(s_{1}\right)<\operatorname{Re}\left(s_{2}\right), \operatorname{Im}\left(s_{1}\right)<\operatorname{Im}\left(s_{2}\right)\right.$

(iv) $\operatorname{Re}\left(s_{1}\right)=\operatorname{Re}\left(s_{2}\right), \operatorname{Im}\left(s_{1}\right)=\operatorname{Im}\left(s_{2}\right)$

Following [15], the authors in [17] developed the notion of complex-valued extended $b$-metric spaces.

Definition 1 (see [17]). Let $\mathfrak{X}$ be a nonempty set and $\xi: \mathfrak{X}$ $\times \mathfrak{X} \longrightarrow[1, \infty)$ be a function. Then, $L_{e}: \mathfrak{X}^{2} \longrightarrow \mathbb{C}$ is known as a complex-valued extended $b$-metric space if the following are satisfied for all $s, \kappa, u \in \mathfrak{X}$ :

(1) $0 \preceq L_{e}(s, \kappa)$ and $L_{e}(s, \kappa)=0$ if and only if $s=\kappa$

(2) $L_{e}(s, \kappa)=L_{e}(\kappa, s)$

(3) $L_{e}(s, \kappa) \preceq \xi(s, \kappa)\left[L_{e}(s, u)+L_{e}(u, \kappa)\right]$

Then, the pair $\left(\mathfrak{X}, L_{e}\right)$ is known as a complex-valued extended $b$-metric space.

As an extension of complex-valued extended $b$-metric spaces, Ullah et al. in [19] introduced the concept of complex-valued rectangular extended $b$-metric spaces.

Definition 2 (see [19]). Let $\mathfrak{X}$ be a nonempty set and $\xi: \mathfrak{X}^{2}$ $\longrightarrow[1, \infty)$ and $L_{r}: \mathfrak{X}^{2} \longrightarrow \mathbb{C}$. We say that $\left(\mathfrak{X}, L_{r}\right)$ is a complex-valued rectangular extended $b$-metric space if for all $a, b \in \mathfrak{X}$ each of which is different from $\kappa, v \in \mathfrak{X}$, we have

(1) $L_{r}(s, \kappa)=0$ if and only if $s=\kappa$

(2) $L_{r}(s, \kappa)=L_{r}(\kappa, s)$

(3) $L_{r}(a, b) \preceq \xi(a, b)\left[L_{r}(a, \kappa)+L_{r}(\kappa, v)+L_{r}(v, b)\right]$

The authors in [20] have recently introduced the idea of triple controlled metric type spaces as follows.

Definition 3 (see [20]). Let $\mathfrak{X}$ be a nonempty set. Given three functions $\xi, \rho, \varsigma: \mathfrak{X}^{2} \longrightarrow[1, \infty)$ and $L_{T}: \mathfrak{X}^{2} \longrightarrow[0, \infty)$. We say that $\left(\mathfrak{X}, L_{T}\right)$ is a triple controlled metric type space if for all $a, b, \kappa, v \in \mathfrak{X}$, we have

(1) $L_{T}(s, \kappa)=0$ if and only if $s=\kappa$

(2) $L_{T}(s, \kappa)=L_{T}(\kappa, s)$

(3) $L_{T}(a, b) \leq \xi(a, \kappa) L_{T}(a, \kappa)+\rho(\kappa, v) L_{T}(\kappa, v)+\varsigma(v, b)$ $L_{T}(v, b)$
Highly motivated by the abovementioned concepts, we now present the definition of complex-valued triple controlled metric spaces.

Definition 4. Let $\mathfrak{X}$ be a nonempty set. Given three functions $\xi, \rho, \varsigma: \mathfrak{X}^{2} \longrightarrow[1, \infty)$ and $L_{t}: \mathfrak{X}^{2} \longrightarrow \mathbb{C}$. We say that $\left(\mathfrak{X}, L_{t}\right)$ is a complex-valued triple controlled metric space if for all $a, b \in \mathfrak{X}$, each of which is different from $\kappa, v \in \mathfrak{X}$, we have

$$
\begin{aligned}
& \text { (1) } L_{t}(s, \kappa)=0 \text { if and only if } s=\kappa \\
& \text { (2) } L_{t}(s, \kappa)=L_{t}(\kappa, s) \\
& \text { (3) } L_{t}(a, b) \leq \xi(a, \kappa) L_{t}(a, \kappa)+\rho(\kappa, v) L_{t}(\kappa, v)+\varsigma(v, b) L_{t}(v \\
& \text {, b) }]
\end{aligned}
$$

Throughout the rest of this paper, we will denote a complex-valued triple controlled metric space by $(\mathrm{CV}$ TCMS). Next, we present the topology of (CV-TCMSs).

Definition 5. Let $\left(\mathfrak{X}, L_{t}\right)$ be a (CV-TCMS).

(1) We say that a sequence $\left\{a_{n}\right\}$ is $L_{t}$-convergent to some $a \in \mathfrak{X}$ if $\left|L_{t}\left(a_{n}, a\right)\right| \longrightarrow 0$ as $n \longrightarrow \infty$

(2) We say that a sequence $\left\{a_{n}\right\}$ is $L_{t}$-Cauchy if and only if $\lim _{n, m \rightarrow \infty}\left|L_{t}\left(a_{n}, a_{m}\right)\right|=0$

(3) We say that $\left(\mathfrak{X}, L_{t}\right)$ is $L_{t}$-complete if for every $L_{t}$-Cauchy sequence is $L_{t}$-convergent

(4) Let $x \in \mathfrak{X}$. An open ball of center $x$ and radius $\eta>0$ in the (CV-TCMS) $\left(\mathfrak{X}, L_{t}\right)$ is $B_{\xi}(x, \eta)=\left\{b \in \mathfrak{X} \mid L_{t}\right.$ $(x, b) \preceq \eta\}$

Note that a CV rectangular metric space is a CV-TCMS. The converse is not true. Next, we present an example that confirms this statement.

Example 1. Let $\mathfrak{X}=\mathfrak{Y} \cup \mathfrak{Z}$ where $\mathfrak{Y}=\{(1 / k) \mid k \in \mathbb{N}\}$ and $\boldsymbol{Z}$ is the set of positive integers. We define $L_{t}: \mathfrak{X}^{2} \longrightarrow \mathbb{C}$ by

$$
L_{t}(a, b)= \begin{cases}0, & \Leftrightarrow a=b, \\ 2 i \beta, & \text { if } a, b \in \mathfrak{V}), \\ \frac{i \beta}{2}, & \text { otherwise, }\end{cases}
$$

where $\beta>0$. Now, define $\xi: \mathfrak{X}^{2} \longrightarrow[1, \infty)$ by $\xi(a, b)=4 \beta$. Given $\rho: \mathfrak{X}^{2} \longrightarrow[1, \infty)$ as $\rho(a, b)=3 \beta$ and $\varsigma: \mathfrak{X}^{2} \longrightarrow[1, \infty)$ as $\varsigma(a, b)=\max \{a, b\}+2 \beta$.

Note that $\left(\mathfrak{X}, L_{t}\right)$ is a CV-TCMS. On the other hand, $\left(\mathfrak{X}, L_{t}\right)$ is not a $\mathrm{CV}$ rectangular metric space. Indeed,

$$
L_{t}\left(\frac{1}{2}, \frac{1}{3}\right)=2 i \beta>L_{t}\left(\frac{1}{2}, 2\right)+L_{t}(2,3)+L_{t}\left(3, \frac{1}{3}\right)=\frac{3 i \beta}{2} \text {. }
$$


In this paper, we prove the Banach and Kannan fixed point results in the setting of CV-TCMSs. Two related applications are also investigated.

\section{Main Results}

Theorem 1. Let $\left(\mathfrak{X}, L_{t}\right)$ be a $L_{t}$-complete CV-TCMS. Let $\mathbb{T}: \mathfrak{X} \longrightarrow \mathfrak{X}$ satisfy $L_{t}(\mathbb{T} x, \mathbb{\mathbb { y }}) \leq \delta L_{t}(x, y)$ where $0<\delta<1$. Assume that there exists $x_{0} \in \mathfrak{X}$ such that the sequence $\left\{x_{n}\right\}$ defined by $x_{n}=\mathbb{T}^{n} x_{0}$ satisfies the following:

$$
\begin{gathered}
\lim _{n \longrightarrow \infty} \xi\left(x_{n}, x_{n+1}\right) \leq \frac{1}{\delta}, \\
\lim _{n \longrightarrow \infty} \rho\left(x_{n}, x_{n+1}\right) \leq \frac{1}{\delta}, \\
\lim _{n \longrightarrow \infty} \varsigma\left(x_{n}, y\right)<\infty, \\
\lim _{n \longrightarrow \infty} \xi\left(y, x_{n}\right)<\infty \text { for any } y \in \mathfrak{X}, \\
\sup _{m \geq 1} \lim _{n \longrightarrow \infty} \xi\left(x_{n}, x_{n+1}\right) \varsigma\left(x_{n}, x_{m}\right) \leq \frac{1}{\delta}, \\
\sup _{m \geq 1} \lim _{n \longrightarrow \infty} \rho\left(x_{n}, x_{n+1}\right) \varsigma\left(x_{n}, x_{m}\right) \leq \frac{1}{\delta} .
\end{gathered}
$$

Then, $\mathbb{T}$ has a unique fixed point in $\mathfrak{X}$.

Proof. First, we have $L_{t}\left(x_{n}, x_{n+1}\right) \leq \delta L_{t}\left(x_{n-1}, x_{n}\right) \leq \delta^{2} L_{t}\left(x_{n-2}\right.$, $\left.x_{n-1}\right) \leq \cdots \leq \delta^{n} L_{t}\left(x_{0}, x_{1}\right)$. Then,

$$
\left|L_{t}\left(x_{n}, x_{n+1}\right)\right| \longrightarrow 0 \text { as } n \longrightarrow \infty \text {. }
$$

Now, let $L_{i}=L_{t}\left(x_{n+i}, x_{n+i+1}\right)$. We need to consider the following two cases.

Case 1. Let $x_{n}=x_{m}$ for some natural numbers $n$ and $m$ with $n \neq m$. Without loss of generality, take $m>n$. If $\mathbb{T}^{m-n}\left(x_{n}\right)=$ $x_{n}$; then, by choosing $y=x_{n}$ and $p=m-n$, we get $\mathbb{T}^{p} y=y$, which implies that $y$ is a periodic point of $\mathbb{T}$. Hence, $L_{t}(y, \mathbb{T}$ $y)=L_{t}\left(\mathbb{T}^{p} y, \mathbb{T}^{p+1} y\right) \leq \delta^{p} L_{t}(y, \mathbb{T} y)$. Since $\delta \in(0,1)$, we get $\mid L_{t}($ $y, \mathbb{T} y) \mid=0$, so $y=\mathbb{T} y$, that is, $\mathbb{T}$ has a fixed point.

From now on, we consider the following case.

Case 2. Assume that for all natural numbers $n \neq m$, we have $x_{n}=\mathbb{T}^{n} x_{0} \neq \mathbb{T}^{m} x_{0}=x_{m}$. Let $n<m$. To prove that $\left\{x_{n}\right\}$ is a $L_{t}$-Cauchy sequence, we need to consider the following two subcases.

Subcase 1. If $m=n+2 p+1$ (where $p \geq 1$ is a fixed natural number), then by the rectangle inequality of the CV-TCMS, we have

$$
\begin{aligned}
& \left|L_{t}\left(x_{n}, x_{n+2 p+1}\right)\right| \leq \xi\left(x_{n}, x_{n+1}\right)\left|L_{t}\left(x_{n}, x_{n+1}\right)\right|+\rho\left(x_{n+1}, x_{n+2}\right) \\
& \text { - } \left.\left|L_{t}\left(x_{n+1}, x_{n+2}\right)\right|+\varsigma\left(x_{n+2}, x_{n+2 p+1}\right)\left|L_{t}\left(x_{n+2}, x_{n+2 p+1}\right)\right|\right] \\
& \leq \xi\left(x_{n}, x_{n+1}\right)\left|L_{t}\left(x_{n}, x_{n+1}\right)\right|+\rho\left(x_{n+1}, x_{n+2}\right)\left|L_{t}\left(x_{n+1}, x_{n+2}\right)\right| \\
& +\varsigma\left(x_{n+2}, x_{n+2 p+1}\right)\left[\xi\left(x_{n+2}, x_{n+3}\right)\left|L_{t}\left(x_{n+2}, x_{n+3}\right)\right|\right. \\
& \cdot+\rho\left(x_{n+3}, x_{n+4}\right)\left|L_{t}\left(x_{n+3}, x_{n+4}\right)\right|+\varsigma\left(x_{n+4}, x_{n+2 p+1}\right) \\
& \text { - } \left.\left|L_{t}\left(x_{n+4}, x_{n+2 p+1}\right)\right|\right] \leq \xi\left(x_{n}, x_{n+1}\right) \delta^{n}\left|L_{0}\right|+\rho\left(x_{n+1}, x_{n+2}\right) \delta^{n+1} \\
& \cdot\left|L_{0}\right|+\varsigma\left(x_{n+2}, x_{n+2 p+1}\right)\left[\xi\left(x_{n+2}, x_{n+3}\right) \delta^{n+2}\left|L_{0}\right|+\rho\left(x_{n+3}, x_{n+4}\right) \delta^{n+3}\right. \\
& \text { - } \left.\left|L_{0}\right|+\varsigma\left(x_{n+4}, x_{n+2 p+1}\right)\left|L_{t}\left(x_{n+4}, x_{n+2 p+1}\right)\right|\right] \leq \xi\left(x_{n}, x_{n+1}\right) \\
& \text { - } \delta^{n}\left|L_{0}\right|+\rho\left(x_{n+1}, x_{n+2}\right) \delta^{n+1}\left|L_{0}\right|+\varsigma\left(x_{n+2}, x_{n+2 p+1}\right) \xi\left(x_{n+2}, x_{n+3}\right) \delta^{n+2} \\
& \text { - }\left|L_{0}\right|+\varsigma\left(x_{n+2}, x_{n+2 p+1}\right) \rho\left(x_{n+3}, x_{n+4}\right) \delta^{n+3}\left|L_{0}\right| \\
& +\varsigma\left(x_{n+2}, x_{n+2 p+1}\right) \varsigma\left(x_{n+4}, x_{n+2 p+1}\right)\left[\xi\left(x_{n+4}, x_{n+5}\right)\left|L_{t}\left(x_{n+4}, x_{n+5}\right)\right|\right. \\
& +\rho\left(x_{n+5}, x_{n+6}\right)\left|L_{t}\left(x_{n+5}, x_{n+6}\right)\right|+\varsigma\left(x_{n+6}, x_{n+2 p+1}\right) \\
& \text { - } \left.\left|L_{t}\left(x_{n+6}, x_{n+2 p+1}\right)\right|\right] \leq \xi\left(x_{n}, x_{n+1}\right) \delta^{n}\left|L_{0}\right|+\rho\left(x_{n+1}, x_{n+2}\right) \delta^{n+1}\left|L_{0}\right| \\
& +\varsigma\left(x_{n+2}, x_{n+2 p+1}\right) \xi\left(x_{n+2}, x_{n+3}\right) \delta^{n+2}\left|L_{0}\right|+\varsigma\left(x_{n+2}, x_{n+2 p+1}\right) \\
& \text { - } \rho\left(x_{n+3}, x_{n+4}\right) \delta^{n+3}\left|L_{0}\right|+\varsigma\left(x_{n+2}, x_{n+2 p+1}\right) \varsigma\left(x_{n+4}, x_{n+2 p+1}\right) \\
& \cdot \xi\left(x_{n+4}, x_{n+5}\right) \delta^{n+4}\left|L_{0}\right|+\varsigma\left(x_{n+2}, x_{n+2 p+1}\right) \varsigma\left(x_{n+4}, x_{n+2 p+1}\right) \\
& \cdot \rho\left(x_{n+5}, x_{n+6}\right) \delta^{n+5}\left|L_{0}\right|+\varsigma\left(x_{n+2}, x_{n+2 p+1}\right) \varsigma\left(x_{n+4}, x_{n+2 p+1}\right) \\
& \cdot \varsigma\left(x_{n+6}, x_{n+2 p+1}\right)\left|L_{t}\left(x_{n+6}, x_{n+2 p+1}\right)\right| \leq \cdots=\xi\left(x_{n}, x_{n+1}\right) \delta^{n}\left|L_{0}\right| \\
& +\rho\left(x_{n+1}, x_{n+2}\right) \delta^{n+1}\left|L_{0}\right|+\sum_{l=1}^{p} \prod_{i=1}^{l} \xi\left(x_{n+2 l}, x_{n+2 l+1}\right) \\
& \cdot \varsigma\left(x_{n+2 i}, x_{n+2 p+1}\right) \delta^{n+2 l} \mid L_{0}+\sum_{l=1}^{p} \prod_{i=1}^{l} \rho\left(x_{n+2 l+1}, x_{n+2 l+2}\right) \\
& \text { · } \varsigma\left(x_{n+2 i}, x_{n+2 p+1}\right) \delta^{n+2 l+1}\left|L_{0}\right| \text {. }
\end{aligned}
$$

Now, given that

$$
\begin{aligned}
& \sup _{m \geq 1} \lim _{n \longrightarrow \infty} \xi\left(x_{n}, x_{n+1}\right) \varsigma\left(x_{n}, x_{m}\right) \leq \frac{1}{\delta}, \\
& \sup _{m \geq 1} \lim _{n \longrightarrow \infty} \rho\left(x_{n}, x_{n+1}\right) \varsigma\left(x_{n}, x_{m}\right) \leq \frac{1}{\delta},
\end{aligned}
$$

we can easily deduce that

$$
\left|L_{t}\left(x_{n}, x_{n+2 p+1}\right)\right| \leq\left[\delta^{n-1}+\delta^{n-1}+\sum_{l=1}^{p} \delta^{n+l}+\sum_{l=1}^{p} \delta^{n+l+1}\right]\left|L_{0}\right| .
$$

Since $\lim _{n \rightarrow \infty} \delta^{n}=0$, the last right-hand side goes to zero at the limit $n \longrightarrow \infty$ (for any integer $p \geq 1$ ). Therefore, $\left\{\left|L_{t}\left(x_{n}, x_{n+2 p+1}\right)\right|\right\}_{n}$ is convergent.

Subcase 2. Let $m=n+2 p$ (where $p \geq 1$ is a fixed integer). First, notice the following:

$L_{t}\left(x_{n}, x_{n+2}\right) \leq \delta L_{\mathrm{t}}\left(x_{n-1}, x_{n+1}\right) \leq \delta^{2} L_{t}\left(x_{n-2}, x_{n}\right) \leq \cdots \leq \delta^{n} L_{t}\left(x_{0}, x_{2}\right)$, 
which leads us to conclude that

$$
\left|L_{t}\left(x_{n}, x_{n+2}\right)\right| \longrightarrow 0 \text { as } n \longrightarrow \infty \text {. }
$$

Thus, by Subcase 1 and using the rectangular inequality of the complex-valued triple controlled metric, we have

$$
\begin{aligned}
& \left|L_{t}\left(x_{n}, x_{n+2 p}\right)\right| \leq \xi\left(x_{n}, x_{n+2 p-3}\right)\left|L_{t}\left(x_{n}, x_{n+2 p-3}\right)\right| \\
& \quad+\rho\left(x_{n+2 p-3}, x_{n+2 p-2}\right)\left|L_{t}\left(x_{n+2 p-3}, x_{n+2 p-2}\right)\right| \\
& \quad+\varsigma\left(x_{n+2 p-2}, x_{n+2 p}\right)\left|L_{t}\left(x_{n+2 p-2}, x_{n+2 p}\right)\right| \leq \xi\left(x_{n}, x_{n+1}\right) \delta^{n}\left|L_{0}\right| \\
& \quad+\rho\left(x_{n+1}, x_{n+2}\right) \delta^{n+1}\left|L_{0}\right|+\sum_{l=1}^{p-1} \prod_{i=1}^{l} \xi\left(x_{n+2 l}, x_{n+2 l+1}\right) \\
& \quad \cdot\left(x_{n+2 i}, x_{n+2 p-3}\right) \delta^{n+2 l}\left|L_{0}\right|+\sum_{l=1}^{p-1} \prod_{i=1}^{l} \rho\left(x_{n+2 l+1}, x_{n+2 l+2}\right) \\
& \quad \varsigma\left(x_{n+2 i}, x_{n+2 p-3}\right) \delta^{n+2 l+1}\left|L_{0}\right|+\varsigma\left(x_{n+2 p-2}, x_{n+2 p}\right) \delta^{n}\left|L_{t}\left(x_{0}, x_{2}\right)\right| .
\end{aligned}
$$

Now, similar to Subcase 1, one can easily deduce that $\left\{\left|L_{\mathrm{t}}\left(x_{n}, x_{n+2 p}\right)\right|\right\}_{n}$ is a convergent sequence as $n \longrightarrow \infty$ (for any integer $p \geq 1$ ). Hence, by Subcases 1 and 2, we conclude that $\left\{x_{n}\right\}$ is a $L_{t}$-Cauchy sequence. Since $\left(\mathfrak{X}, L_{t}\right)$ is a $L_{t}$ -complete CV-TCMS, there is $v \in \mathfrak{X}$ such that $\left\{x_{n}\right\} \longrightarrow v$ as $n \longrightarrow \infty$.

Now, if there exists $N \in \mathbb{N}$ such that $x_{N}=v$, then since we deal with Case 2, one writes $x_{n}=\mathbb{T}^{n} x_{0} \neq v$ for all $n>N$. Also, $x_{n}=\mathbb{T}^{n} x_{0} \neq \mathbb{V} v$ for all $n>N$. Next, assume that there exists $N \in \mathbb{N}$ with $x_{N}=\mathbb{T}^{N} x_{0}=\mathbb{} v$. Once again, we confirm that $x_{n}=\mathbb{T}^{n} x_{0} \in\{v, \mathbb{v} v\}$ for all $n>N$. Thus, without loss of generality, we may assume $x_{n} \in\{v, \mathbb{v}\}$ for all natural numbers $n$. We have

$$
\begin{aligned}
& L_{t}(v, \mathbb{v}) \preceq \xi\left(\nu, x_{n}\right) L_{t}\left(\nu, x_{n}\right)+\rho\left(x_{n}, x_{n+1}\right) L_{t}\left(x_{n}, x_{n+1}\right) \\
& +\varsigma\left(x_{n+1}, \mathbb{T} v\right) L_{t}\left(x_{n+1}, \mathbb{\nabla} v\right) \preceq \xi\left(v, x_{n}\right) L_{t}\left(v, x_{n}\right) \\
& +\rho\left(x_{n}, x_{n+1}\right) L_{t}\left(x_{n}, x_{n+1}+\varsigma\left(x_{n+1}, \mathbb{v}\right) \delta L_{t}\left(x_{n}, v\right)\right. \text {, }
\end{aligned}
$$

which implies

$$
\begin{gathered}
\left|L_{t}(v, \mathbb{\nabla})\right| \leq \xi\left(v, x_{n}\right)\left|L_{t}\left(v, x_{n}\right)\right|+\rho\left(x_{n}, x_{n+1}\right)\left|L_{t}\left(x_{n}, x_{n+1}\right)\right| \\
+\varsigma\left(x_{n+1}, \mathbb{\nabla} v\right) \delta\left|L_{t}\left(x_{n}, v\right)\right| .
\end{gathered}
$$

Therefore, in view of the assumptions in the theorem, as $n \longrightarrow \infty$, we deduce that $\left|L_{t}(v, \mathbb{v})\right|=0$ and that is $\mathbb{v} v=v$ as required.

In closing, assume there exist two fixed points of $\mathbb{T}$, say $v$ and $\mu$ where $v \neq \mu$. Thus,

$$
L_{t}(\nu, \mu)=L_{t}(\mathbb{v} v, \mathbb{T} \mu) \preceq \delta L_{t}(\nu, \mu) \prec L_{t}(\nu, \mu),
$$

which is a contradiction. Therefore, the fixed point of $\mathbb{T}$ is unique.
Theorem 2. Let $\left(\mathfrak{X}, L_{t}\right)$ be a $L_{t}$-complete $C V$-TCMS and $\mathbb{T}$ be a self mapping on $\mathfrak{X}$ satisfying the following condition: for all $a, b \in \mathfrak{X}$, there exists $0<\delta<1 / 2$ such that

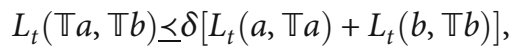

and there exists $x_{0} \in \mathfrak{X}$ in order that the sequence $\left\{x_{n}\right\}$ defined by $x_{n}=\mathbb{T}^{n} x_{0}$ satisfies the following:

$$
\begin{gathered}
\lim _{n \longrightarrow \infty} \xi\left(y, x_{n}\right) \leq \frac{1}{\delta}, \\
\lim _{n \longrightarrow \infty} \varsigma\left(x_{n}, y\right)<\frac{1}{\delta} \quad \text { for any } y \in \mathfrak{X}, \\
\lim _{n \longrightarrow \infty} \rho\left(x_{n}, x_{n+1}\right) \leq \frac{1}{\delta} .
\end{gathered}
$$

Then, $\mathbb{T}$ has a unique fixed point in $\mathfrak{X}$.

Proof. First of all, note that for all $n \geq 1$, we have

$$
L_{t}\left(x_{n}, x_{n+1}\right) \leq \delta\left[L_{t}\left(x_{n-1}, x_{n}\right)+L_{t}\left(x_{n}, x_{n+1}\right)\right] \text {. }
$$

Consequently,

$$
L_{t}\left(x_{n}, x_{n+1}\right) \preceq \frac{\delta}{1-\delta} L_{t}\left(x_{n-1}, x_{n}\right)
$$

Since $0<\delta<1 / 2$, one has $0<(\delta /(1-\delta))<1$. Set $\mu=\delta /$ $(1-\delta)$. One writes

$$
\begin{aligned}
\left|L_{t}\left(x_{n}, x_{n+1}\right)\right| & \leq \mu\left|L_{t}\left(x_{n-1}, x_{n}\right)\right| \leq \mu^{2}\left|L_{t}\left(x_{n-2}, x_{n-1}\right)\right| \\
& \leq \cdots \leq \mu^{n}\left|L_{t}\left(x_{0}, x_{1}\right)\right| .
\end{aligned}
$$

Therefore,

$$
\left|L_{t}\left(x_{n}, x_{n+1}\right)\right| \longrightarrow 0 \text { as } n \longrightarrow \infty \text {. }
$$

Also, for all $n, m \geq 1$, we have

$$
L_{t}\left(x_{n}, x_{m}\right) \leq \delta\left[L_{t}\left(x_{n-1}, x_{n}\right)+L_{t}\left(x_{m-1}, x_{m}\right)\right] .
$$

By (19), we deduce that $\left|L_{t}\left(x_{n}, x_{m}\right)\right| \longrightarrow 0$ as $n, m \longrightarrow \infty$. Hence, $\left\{x_{n}\right\}$ is a $L_{t}$-Cauchy sequence. Since $\left(\mathfrak{X}, L_{t}\right)$ is a $L_{t}$-complete CV-TCMS, the sequence $\left\{x_{n}\right\}$ converges to some $v \in \mathfrak{X}$.

By the argument of the proof of Theorem 1, assume that for all $n \geq 1$, we have $x_{n} \in\{v, \mathbb{v} v\}$. Thus,

$$
\begin{aligned}
& L_{t}(v, \mathbb{v}) \preceq \xi\left(v, x_{n}\right) L_{t}\left(v, x_{n}\right)+\rho\left(x_{n}, x_{n+1}\right) L_{t}\left(x_{n}, x_{n+1}\right) \\
& +\varsigma\left(x_{n+1}, \mathbb{\nabla} v\right) L_{t}\left(x_{n+1}, \mathbb{\nabla} v\right) \preceq \xi\left(v, x_{n}\right) L_{t}\left(v, x_{n}\right) \\
& +\rho\left(x_{n}, x_{n+1}\right) L_{t}\left(x_{n}, x_{n+1}\right)+\varsigma\left(x_{n+1}, \mathbb{v} v\right) \\
& \cdot \delta\left[L_{t}\left(x_{n}, \mathbb{} x_{n}\right)+L_{t}(v, \mathbb{v})\right] \text {. }
\end{aligned}
$$


As $n \longrightarrow \infty$, we obtain

$$
\begin{aligned}
\left|L_{t}(v, \mathbb{} v)\right| & \leq 0+0+0+\underset{n \longrightarrow \infty}{\lim \sup } \varsigma\left(x_{n+1}, \mathbb{} v\right) \delta\left|L_{t}(v, \mathbb{} v)\right| \\
& <\left|L_{t}(v, \mathbb{} v)\right|
\end{aligned}
$$

At the limit $n \longrightarrow \infty$, we find that $\left|L_{t}(v, \mathbb{v})\right|=0$ and that is $\mathbb{v}=v$ as required. Now, assume that we have two fixed points of $\mathbb{T}$, say $v$ and $s$. Therefore,

$$
\left|L_{t}(\nu, s)\right|=\left|L_{t}(\mathbb{T} v, \mathbb{T} s)\right| \leq \delta\left(\left|L_{t}(\nu, v)\right|+\left|L_{t}(s, s)\right|\right)=0
$$

Hence, $v=s$, as desired.

\section{Applications}

4.1. A Fredholm-Type Integral Equation. Consider the set $\mathfrak{X}$ $=C([0,1], \mathbb{R})$. Given the following Fredholm-type integral equation

$$
a^{\prime}(t)=\int_{0}^{1} \mathbb{M}\left(t, s, a^{\prime}(t)\right) d s, \quad \text { for } t, s \in[0,1]
$$

where $\mathbb{M}\left(t, s, a^{\prime}(t)\right)$ is a continuous function from $[0,1]^{2}$ into $\mathbb{R}$. Now, define

$$
\begin{array}{rc}
L_{t}: \mathfrak{X} & \times \mathfrak{X} \longrightarrow \mathbb{C} \\
(a, b) \mapsto i \sup _{t \in[0,1]}\left(\frac{|a(t)|+|b(t)|}{2}\right) .
\end{array}
$$

Note that $\left(\mathfrak{X}, L_{t}\right)$ is a complete CV-TCMS, where

$$
\xi(a, b)=2, \rho(a, b)=1 \text { and } \varsigma(a, b)=3 \text {. }
$$

Theorem 3. Assume that for all $a, b \in \mathfrak{X}$

(1) $\left|\mathbb{M}\left(t, s, a^{\prime}(t)\right)\right|+|\mathbb{M}(t, s, b(t))| \leq \delta\left(\left|a^{\prime}(t)\right|+|b(t)|\right)$, for some $\delta \in[0,1 / 4)$

(2) $\mathbb{M}\left(t, s, \int_{0}^{1} \mathbb{M}\left(t, s, a^{\prime}(t)\right) d s\right)<\mathbb{M}\left(t, s, a^{\prime}(t)\right)$ for all $t, s$

Then, the above integral equation has a unique solution.

Proof. Let $\mathbb{T}: \mathfrak{X} \longrightarrow \mathfrak{X}$ be defined by $\mathbb{T} a^{\prime}(t)=\int_{0}^{1} \mathbb{M}\left(t, s, a^{\prime}\right.$ $(t)) d s$. Then,

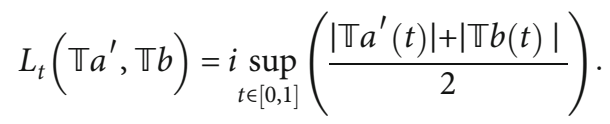

Now, we have

$$
\begin{aligned}
L_{t}\left(\mathbb{T} a^{\prime}(t), \mathbb{T} b(t)\right) & =i \frac{\left|\mathbb{T} a^{\prime}(t)\right|+|\mathbb{T} b(t)|}{2} \\
& =i \frac{\left|\int_{0}^{1} \mathbb{M}\left(t, s, a^{\prime}(t)\right) d s\right|+\left|\int_{0}^{1} \mathbb{M}(t, s, b(t)) d s\right|}{2} \\
& \leq i \frac{\int_{0}^{1}\left|\mathbb{M}\left(t, s, a^{\prime}(t)\right)\right| d s+\int_{0}^{1}|\mathbb{M}(t, s, b(t))| d s}{2} \\
& =i \frac{\int_{0}^{1}\left(\left|\mathbb{M}\left(t, s, a^{\prime}(t)\right)\right|+|\mathbb{M}(t, s, b(t))|\right) d s}{2} \\
& \leq i \frac{\int_{0}^{1} \delta\left(\left|a^{\prime}(t)\right|+|b(t)|\right) d s}{2} \\
& \leq \delta L_{t}\left(a^{\prime}(t), b(t)\right) .
\end{aligned}
$$

Thus, $L_{t}\left(\mathbb{T} a^{\prime}, \mathbb{}(b) \leq \delta L_{t}\left(a^{\prime}, b\right)\right.$. Since $\delta \in[0,1 / 4)$, one gets

$$
\begin{aligned}
\xi(a, b)<\frac{1}{\delta} \\
\rho(a, b)<\frac{1}{\delta} \\
\varsigma(a, b)<\frac{1}{\delta} .
\end{aligned}
$$

Therefore, all the hypotheses of Theorem 1 are satisfied, and hence, equation (24) has a unique solution.

4.2. A Polynomial Equation of a Degree Greater or Equal to 3. The following is an application on higher degree polynomial equations.

Theorem 4. For any natural number $\beta \geq 3$ and real $|\alpha| \leq 1$, the following equation

$$
\alpha^{\beta}+1=\left(\beta^{4}-1\right) \alpha^{\beta+1}+\beta^{4} \alpha
$$

has a unique real solution.

Proof. It is not difficult to see that if $|\alpha|>1$, equation (30) does not have a solution. So, let $\mathfrak{X}=[-1,1]$ and for all $\alpha, r$ $\in \mathfrak{X}$, let $L_{t}(\alpha, r)=|\alpha-r|+i|\alpha-r|$ and $\xi(u, v)=3, \rho(u, v)$ $=4$ and $\varsigma(u, v)=\max \{u, v\}+2$. Note that $\left(\mathfrak{X}, L_{t}\right)$ is a $L_{t}$ -complete CV-TCMS. Now, let

$$
\mathbb{} \alpha=\frac{\alpha^{\beta}+1}{\left(\beta^{4}-1\right) \alpha^{\beta}+\beta^{4}} .
$$


Notice that, since $\beta \geq 2$, we can deduce that $\beta^{4} \geq 6$. Thus,

$$
\begin{aligned}
L_{t}(\mathbb{T} \alpha, \mathbb{} r)= & \left|\frac{\alpha^{\beta}+1}{\left(\beta^{4}-1\right) \alpha^{\beta}+\beta^{4}}-\frac{r^{\beta}+1}{\left(\beta^{4}-1\right) r^{\beta}+\beta^{4}}\right| \\
& +i\left|\frac{\alpha^{\beta}+1}{\left(\beta^{4}-1\right) \alpha^{\beta}+\beta^{4}}-\frac{r^{\beta}+1}{\left(\beta^{4}-1\right) r^{\beta}+\beta^{4}}\right| \\
= & \left|\frac{\alpha^{\beta}-r^{\beta}}{\left(\left(\beta^{4}-1\right) \alpha^{\beta}+\beta^{4}\right)\left(\left(\beta^{4}-1\right) r^{\beta}+\beta^{4}\right)}\right| \\
& +i\left|\frac{\alpha^{\beta}-r^{\beta}}{\left(\left(\beta^{4}-1\right) \alpha^{\beta}+\beta^{4}\right)\left(\left(\beta^{4}-1\right) r^{\beta}+\beta^{4}\right)}\right| \\
\leq & \frac{|\alpha-r|}{\beta^{4}+i \frac{|\alpha-r|}{\beta^{4}} \leq \frac{|\alpha-r|}{6}+i \frac{|\alpha-r|}{6}} \\
= & \frac{1}{6} L_{t}(\alpha, r) .
\end{aligned}
$$

Hence,

$$
L_{t}(\mathbb{T} \alpha, \mathbb{} r) \leq \delta L_{t}(\alpha, r), \quad \text { where } \delta=\frac{1}{6}
$$

Moreover, it is easy to see that for all $\alpha_{0} \in \mathfrak{X}$, we have

$$
\alpha_{n}=\mathbb{T}^{n} \alpha_{0} \leq \frac{2}{\beta^{4}} .
$$

Note that all the conditions of Theorem 1 are satisfied. Thus, $\mathbb{T}$ possesses a unique fixed point in $\mathfrak{X}$, and equation (30) has a unique real solution.

\section{Conclusion}

Finally, we would like to leave the following questions.

Question 1. Let $\left(\mathfrak{X}, L_{t}\right)$ be a CV-TCMS and $\mathbb{T}: \mathfrak{X} \longrightarrow \mathfrak{X}$. Given a function $\varsigma: \mathfrak{X}^{2} \longrightarrow[1, \infty)$. Suppose there exists $\delta \in$ $(0,1)$ such that, for all $s, r \in \mathfrak{X}$,

$$
L_{t}\left(\mathbb{T} s, \mathbb{}[r) \leq \delta \varsigma(s, r) L_{t}(s, r)\right.
$$
in $\mathfrak{X}$ ?

Under what conditions does $\mathbb{T}$ have a unique fixed point

Question 2. Let $\left(\mathfrak{X}, L_{t}\right)$ be a CV-TCMS, and $\mathbb{T}: \mathfrak{X} \longrightarrow \mathfrak{X}$. Given a function $\varsigma: \mathfrak{X}^{2} \longrightarrow[1, \infty)$. Suppose there exists $\delta \in$ $(0,1 / 2)$ such that, for all $s, r \in \mathfrak{X}$,

$$
L_{t}(\mathbb{T} s, \mathbb{T} r) \preceq \delta \varsigma(s, r)\left[L_{t}(s, \mathbb{T} s)+L_{t}(r, \mathbb{T} r)\right]
$$

Under what conditions does $\mathbb{T}$ have a unique fixed point in $\mathfrak{X}$ ?

\section{Data Availability}

Data sharing is not applicable to this article as no data set were generated or analyzed during the current study.

\section{Conflicts of Interest}

The authors declare no conflict of interest.

\section{Authors' Contributions}

All the authors have equally contributed to the final manuscript.

\section{Acknowledgments}

The authors would like to thank Prince Sultan University for funding this work through research group Nonlinear Analysis Methods in Applied Mathematics (NAMAM) group number RG-DES-2017-01-17.

\section{References}

[1] S. Banach, "Sur les opérations dans les ensembles abstraits et leur application aux équations intégrales," Fundamenta Mathematicae, vol. 3, no. 1, pp. 133-181, 1922.

[2] M. Aslantas, H. Sahin, and D. Turkoglu, "Some Caristi type fixed point theorems," The Journal of Analysis, vol. 29, no. 1, pp. 89-103, 2020.

[3] H. Sahin, M. Aslantas, and I. Altun, "Feng-Liu type approach to best proximity point results for multivalued mappings," Journal of Fixed Point Theory and Applications, vol. 22, no. 1, p. 11, 2020.

[4] P. Patle, D. Patel, H. Aydi, and S. Radenovic, "On $\mathrm{H}^{+}$-type multivalued contractions and applications in symmetric and probabilistic spaces," Mathematics, vol. 7, no. 2, p. 144, 2019.

[5] I. Altun, M. Aslantas, and H. Sahin, "Best proximity point results for p-proximal contractions," Acta Math. Hungar, vol. 162 , no. 2 , pp. 393-402, 2020.

[6] M. Aslantas, H. Sahin, and I. Altun, "Best proximity point theorems for cyclic p-contractions with some consequences and applications," Nonlinear Analysis: Modelling and Control, vol. 26, no. 1, pp. 113-129, 2021.

[7] E. Karapinar, S. Czerwik, and H. Aydi, "Meir-Keeler contraction mappings in generalized -metric spaces," Journal of Function spaces, vol. 2018, Article ID 3264620, 4 pages, 2018.

[8] H. Aydi, E. Karapinar, and A. Roldán López de Hierro, “ $\omega$ Interpolative Ćirić-Reich-Rus-type contractions," Mathematics, vol. 7, no. 1, p. 57, 2019.

[9] N. Saleem, J. Vujaković, W. U. Baloch, and S. Radenović, "Coincidence point results for multivalued Suzuki type mappings using $\theta$-contraction in $b$-metric spaces," Mathematics, vol. 7, no. 11, p. 1017, 2019.

[10] N. Saleem, B. Ali, Z. Raza, and M. Abbas, "Fixed points of Suzuki-type generalized multivalued $(f, \theta, L)$ almost contractions with applications," Filomat, vol. 33, no. 2, pp. 499-518, 2019.

[11] I. A. Bakhtin, "The contraction mapping principle in almost metric spaces,” Functional Analysis, vol. 30, pp. 26-37, 2020. 
[12] S. Czerwik, "Contraction mappings in b-metric spaces," Acta Mathematica et Informatica Universitatis Ostraviensis, vol. 1, pp. 5-11, 1993.

[13] T. Abdeljawad, N. Mlaiki, H. Aydi, and N. Souayah, "Double controlled metric type spaces and some fixed point results," Mathematics, vol. 6, no. 12, p. 320, 2018.

[14] N. Mlaiki, H. Aydi, N. Souayah, and T. Abdeljawad, "Controlled metric type spaces and the related contraction principle," Mathematics, vol. 6, no. 10, p. 194, 2018.

[15] A. Azam, B. Fisher, and M. Khan, "Common fixed point theorems in complex valued metric spaces," Numerical Functional Analysis and Optimization, vol. 32, no. 3, pp. 243-253, 2011.

[16] K. P. R. Rao, P. R. Swamy, and J. R. Prasad, "A common fixed point theorem in complex valued $b$-metric spaces," Bulletin of Mathematics and Statistics Research, vol. 1, pp. 1-8, 2013.

[17] N. Ullah, M. S. Shagari, and A. Azam, "Fixed point theorems in complex valued extended $b$-metric spaces," Moroccan Journal of Pure and Applied Analysis, vol. 5, no. 2, pp. 140-163, 2019.

[18] J. Rezaei Roshan, V. Parvaneh, S. Sedghi, N. Shobkolaei, and W. Shatanawi, "Common fixed points of almost generalized $(\psi, \varphi)_{s^{-}}$contractive mappings in ordered b-metric spaces," Fixed Point Theory and Applications, vol. 2013, no. 1, Article ID 159, 2013.

[19] N. Ullah and M. S. Shagari, "Fixed point results in complex valued rectangular extended $b$-metric spaces with applications," Mathematical Analysis \& Complex Optimization, vol. 1, no. 2, pp. 107-120, 2020.

[20] S. T. Z, K. Gopalan, and T. Abdeljawad, "A different approach to fixed point theorems on triple controlled metric type spaces with a numerical experiment," Dynamic Systems and Applications, vol. 30, no. 1, pp. 111-130, 2021. 